\title{
PLAN DE REPOSICIONAMIENTO DEL DESTINO TURÍSTICO DE SAN LUIS, ARGENTINA
}

\author{
Equipo de Máster Oficial en \\ Planificación y Dirección del turismo \\ Promoción de productos turísticos ${ }^{1}$
}

Material original autorizado para su primera publicación en la revista académica REDMARKA. Revista Digital de Marketing Aplicado.

https://doi.org/10.17979/redma.2011.01.06.4727

\section{RESUMEN}

Hoy en día existen muchos países que se reconocen a nivel mundial por sus avances y desarrollos tecnológicos. Por esto y por su importancia actual, este proyecto intenta sacarle provecho a todas las bondades de la tecnología (turismo cinematográfico y música, entre otros) creando así una nueva modalidad de turismo que nos ayude a cumplir el objetivo principal de este trabajo que es posicionar el destino San Luis, ubicado en la región centro de Argentina.

Se elaboraron una serie de estrategias a corto, medio y largo plazo, las cuales pudimos determinar gracias al análisis, estudio y evaluación previos realizados de la situación con que contaba la provincia y del nuevo producto a explotar.

\footnotetext{
${ }^{1}$ Equipo del proyecto:

Máster Oficial en Planificación y Dirección del turismo

Promoción de productos turísticos

Alba Fernández Arcos albafarcos@gmail.com

Carolina Cardoso Rolo@karolcarolina_@ hotmail.com 1149

Ester López González $\quad$ eslogon19@gmail.com 1150

Juan Pablo Falcón juanpablofalcon@hotmail.com 1151

Mavis Anet Forbes Gudiño mavita42@hotmail.com 1152

Míriam Sánchez Canedo mirisc@hotmail.es 1153

Soledad Pardo Neira soledadcarreras@ymail.com 1154

Susana Trigo Rossier $\quad$ susanatr84@hotmail.com 1155

Diseño gráfico:

Noemi Sánchez Canedo noemisc27@gmail.com 1156

Profesores tutores del proyecto:

Valentín Alejandro Martínez Fernández

Magdalena Rodríguez Fernández
} 
Dada la importancia e impacto que tiene la marca a la hora de promocionar un destino, fue fundamental crear una para San Luis con el fin de alcanzar nuestro objetivo principal de posicionar esta provincia y darla a conocer.

Palabras claves: turismo tecnológico, posicionamiento, turismo cinematográfico, marca de destino.

\author{
ABSTRACT \\ PLANNING FOR THE REPOSITIONING OF THE TOURISM DESTINATION SAN LUIS, \\ ARGENTINA
}

Nowadays, many countries worldwide are known by their advances and technology developments. Owing to this fact and the relevance it means today, this project is about taking advantages from all the benefits of technologies (music and cinematographic tourism, among others), in order to create a new kind of tourism that help us make possible the main object of this work: the repositioning of San Luis, an interesting central region of Argentina.

A sequence of short, medium and long term strategies were devised, which could be determined as a result of the previous analysis, studies and evaluation of the condition of the province and the new product to exploit.

Keywords: Technologic tourism, positioning, cinematographic tourism, tourism brand 


\section{Introducción}

El presente proyecto consiste en el plan para posicionar la provincia de San Luis ubicada en la región centro de Argentina. Para ello se realizó un análisis de su imagen nacional e internacional, esto pudo ser posible mediante un proceso de encuestas físicas (España) y online( Argentina), con el fin de determinar cuál era la percepción, valoración y nivel de conocimiento sobre este destino; al mismo tiempo se estaba llevando a cabo un análisis de inventario de sus recursos: naturales, hidrográficos, histórico-culturales, fiestas y folklore, ocio y tecnologías, económicos e infraestructura, que permitió conocer la situación actual y con que cuenta realmente la provincia para ofrecer como destino.

Por el impacto del internet en el sector turismo y la fuerza que ejerce para atraer posibles turistas o visitantes a un destino, se evaluó su posicionamiento en la web e internet; también fue necesario el estudio de su competencia directa: Mendoza, San Juan, y Córdoba que junto la matriz nos permitió identificar similitudes y diferencias; además se elaboro un análisis DAFO que nos fue de gran ayuda a la hora de construir las estrategias para este proyecto.

Después de analizar, estudiar y evaluar San Luis, estamos convencidos de que puede presumir como uno de los pocos destinos que cuentan con una rica diversidad de atractivos para ofrecer. Éste proyecto busca diferenciarlo de los demás destinos a través de su ventaja competitiva más notable: un turismo de tecnología "música y cine" que pueda entrelazar cada uno de sus recursos con esta nueva modalidad. Creemos que la tecnología ya no es más un tema del futuro, si no del presente y que permite la oportunidad para fidelizar y atraer nuevos turistas siendo una ventaja sostenible que puede perdurar en el tiempo.

Al encontrar el producto que nos ayudaría a posicionar San Luis se realizaron nuevos estudios de otras experiencias de cine en el mundo, como sacaban provecho de ella, cuáles y porque eran las más importantes; la infraestructura en un lugar que apuesta por la tecnología jugaba un papel de suma importancia, y esto fue lo que nos animo a buscar información sobre los hoteles 
y restaurante Geek (término que se utiliza para referirse a las personas fascinadas por la tecnología e informática).

Por último y no por eso menos importante se realizó con la ayuda de una especialista en diseño, la marca turística para San Luis; la cual vimos necesaria para lograr nuestro objetivo de posicionar el destino. Todos los estudios ya mencionados, test en los artículos y productos que queremos promocionar, permitieron una evolución de la marca para al fin escoger la que representaría a la provincia.

\section{Ventajas competitivas de San Luis}

- Industria cinematográfica, festival internacional de cine San Luis

- Desarrollo tecnológico y apoyo del ministerio y auge de nuevas ciudades modernas, La Punta

- $\quad$ Recursos naturales aptos para la práctica de turismo alternativo.

- $\quad$ Autenticidad de los pueblos así como su cultura.

\section{Proceso de creación del diseño de producto}

Tras el oportuno inventario de recursos turísticos hemos concluido que la ventaja competitiva más saliente de San Luis y que sin duda lo diferencia de la competencia es la industria cinematográfica que se desarrolla en la provincia.

Aprovechando el auge en desarrollo de nuevas tecnologías que experimenta San Luis combinado con el auge de su industria cinematográfica pero sin olvidar los parajes naturales que presenta, resulta interesante esta combinación para transmitir una nueva imagen de San Luis.

La política que sigue el gobierno de San Luis con respecto a la inversión tecnológica y la industria del cine es claramente favorable. Está claro que San Luis pretende destacar en este aspecto por lo que la coherencia de la propuesta es evidente.

La infraestructura y equipamientos con respecto a la industria cinematográfica de San Luis ya existen solo se trata de crear otros usos a través del turismo. 
Existe un programa muy interesante que parte del Ministerio de Turismo, Cultura y Deporte llamado San Luis Cine (programa de Cine y Música).

El cine es un buen instrumento de promoción y a través del cual resulta efectivo llegar hasta nuestro público objetivo. Además resulta una herramienta muy creativa y presenta un abanico amplio de posibilidades a la hora de utilizarlo para la promoción de un destino.

\section{Clasificación de destinos turísticos objeto de esta actividad}

1. Localizaciones de rodaje de producciones cinematográficas

2. Lugares donde se desarrollada la trama de una película

3. Ciudades donde se ubican grandes estudios cinematográficos

4. Poblaciones ligadas la vida de actores, productores, guionistas o directores.

5. Museos y otros recursos

Definición de turismo cinematográfico, movie tourism, film commission. Fuente hosteltur.

TURISMO CINEMATOGRÁFICO: Turismo relacionado con el cine. Este concepto engloba tanto los viajes motivados por una película, como la promoción de los destinos a través del cine.

MOVIE TOURISM: Turismo inducido por el cine. Por un lado, se aprovecha el interés por visitar las localizaciones de los rodajes y por otro se utilizan las películas como escaparate de los atractivos turísticos.

FILM COMMISSION: Organismo público, sin ánimo de lucro, encargado de conseguir que su zona geográfica de influencia sea el escenario del mayor número de rodajes posible.

Si su ámbito de actuación es una región, su nombre es film commission. Si se circunscribe a una localidad, habitualmente se denomina film office. A pesar de las diferentes nomenclaturas, sus funciones son las mismas. Promocionan el territorio en el que trabajan para que las productoras se decanten por él para 
sus rodajes y responden a la demanda de aquellas que solicitan información cuando aún no han decidido dónde filmar.

\section{El nuevo producto turístico}

Se pretende crear un producto específico basado en la tecnología y la cultura proyectada a través de la industria cinematografica, en la cual el consumidor turístico pueda satisfacer su inquietud viajera en todo el territorio de San Luis mediante la lente del cineasta.

Con la industria cinematográfica pretendemos crear un producto que atiende a una clara segmentación del mercado pero con posibilidades de ampliarse, se trata del turismo cinematográfico. Este tipo de turismo se define por aquellas personas interesadas o amantes del cine (aunque dentro de la industria del cine podemos distinguir varias modalidades o categorías, por ahora nos vamos a referir al cine en general). El turismo cinematográfico involucra a muchos agentes y variedad de público. Por un lado, el consumidor puede estar interesado en una simple producción que se haya realizado en San Luis, puede interesarle todos los aspectos del rodaje, la producción, interpretación, dirección...e incluso tomar parte en el proceso o sencillamente aprender algunas técnicas en la filmografía o interpretación, por otro lado, puede ser un consumidor ya experimentado que recurre a San Luis para mejorar su técnica por ejemplo. Pero también hemos planteado la circunstancia de que el turista en potencia no tenga un interés explícito en el cine pero sin embargo decida aumentar su estancia ante la novedad del producto y así comenzaremos a fidelizar a nuestro cliente.

$>\quad$ La idea de los movie maps y sistemas de localización terrestres (GPS) para situar escenarios naturales.

$>\quad$ El cine móvil para llegar a esas escenas naturales.

$>\quad$ Utilización de instalaciones y equipamientos: Set cine San Luis. 


\begin{tabular}{l}
\hline$\quad$ Talleres y cursos para los turistas interesados. / Alquiler de equipo \\
$>\quad$ Monitores de apoyo y ayuda. \\
méritos: realizando un concurso o feria donde todos los turistas que hayan \\
participado en la experiencia puedan exponer sus videos. \\
$>\quad$ La Web 2.0 para compartir experiencias y videos, fidelizar y captar \\
consumidores. \\
$>\quad$ La herramienta youtube como medio de difusión \\
$>\quad$ La trayectoria cinematográfica de San Luis y el Bicentenario. \\
$>\quad$ Relación con otros festivales internacionales
\end{tabular}

Lo novedoso del producto de turismo cinematográfico reside en el mero hecho de poner al alcance de la población que lo desee o con la capacidad adquisitiva adecuada el uso y disfrute de una experiencia cinematográfica. Para ello nuestro principal telón de entrada es el festival de cine internacional (como acto inaugural del nuevo producto turístico, por ejemplo). Aunque sin duda, no podemos olvidar los parajes naturales y culturales de la provincia que conformarán el escenario de este turismo cinematográfico. Se trata de disfrutar y estudiar la naturaleza desde la perspectiva del cine. Deberá ser un uso totalmente sostenible pues el escenario siempre es el mismo, San Luis, y ello debe ayudar a valorar la naturaleza y comprender la importancia de su existencia incluso para el cine. La nuevas tecnologías también estarán presentes pues recordemos que el cine se está reinventando e innovando en todos sus campos, por ejemplo el cine en 3D: (mostrando al público como se rueda una película en 3D por ejemplo)

\section{La Marca del destino turístico}

\section{Diseño de la marca turística para San Luis, Argentina.}


Con el objetivo de posicionar el destino San Luis se ha establecido como una estrategia prioritaria y de espacial relevancia dotar a San Luis con una marca turística a través de la cual podamos llegar al posicionamiento que deseamos.

Entendemos por marca un nombre y/o símbolo distintivo (logotipo, marca registrada o diseño) que sirve para identificar los productos o servicios de un fabricante o grupo de fabricantes y para diferenciar aquellos productos 0 servicios de los de su competencia (Aaker, D.A.,1994). Pero además de su identidad, una marca tiene una reputación construida en base a su promesa y desempeño.

A través de la marca turística bien posicionada, se podrá generar una gran cantidad de recursos en beneficio de los prestadores de servicios del sector turismo no solo a través de publicidad, sino con la venta de todo tipo de mercaderías como: folletos, camisetas, gorras, tazas, pósters, muñecos, relojes, collares, joyas, calendarios, libretas, lápices, plumas, maletas, página web, etc. La marca como certificadora de prestadores de servicios o de elaboración de productos, va por lo general relacionada con un estándar de calidad, y el uso puede prohibirse o limitarse dependiendo del grado de cumplimiento de esos estándares, lo que sin duda beneficia no solo a los prestadores de servicios certificados con la marca, sino también a los visitantes o consumidores, y por supuesto, a la administración gubernamental encargada del manejo de la marca.

Pero además pretendemos ir más allá con los usos de la marca creando una "marca paraguas", es decir, una única marca de la provincia que aparezca plasmada en todos los productos alimenticios que se envasen y comercialicen en San Luis en concreto en Villa Mercedes que es donde se sitúa toda la industria productora de la provincia.

Pensar en una región como una marca trae el desafío de destacarse, llamar la atención, y transmitir una promesa o intención clara. De este modo, se llegará a través de esta vía hacia una buena ventaja competitiva.

Hasta el momento el turista que llega a San Luis es un visitante casual o de paso por ello esta nueva marca deberá ser impactante y evocadora. 
En San Luis cada quien delinea un recorrido personal, diferente, que le permite adentrarse, descubrir, cultivarse, vivir y entender que San Luis es acción. La experiencia de San Luis es tan particular e individual que además de los intereses inicial que un turista pueda tener. Lo realmente importante es suscitar nuevos intereses, deseos y pasiones. El objetivo es crear ese deseo de volver, de modo que cada vez que se repita se pueda hacer por diferentes motivaciones.

El turismo polifacético de San Luis y en muchas ocasiones relacionado con un turismo activo nos permitió englobar todas sus dimensiones bajo la mirada del cine y el desarrollo tecnológico.

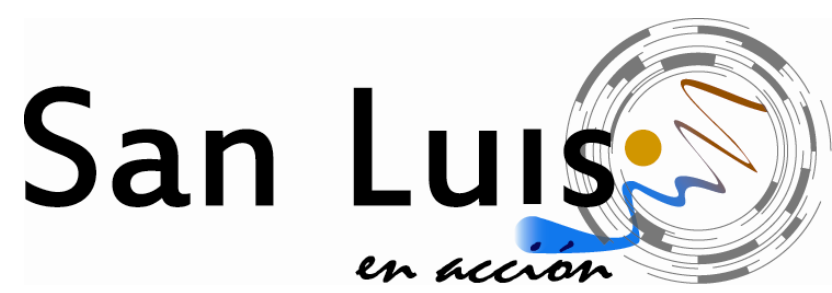

Ecuación de la marca:

Paisaje (montañas, ríos y lagos) + microclima + cine + tecnología= ARTE= San Luis

Componentes de la marca

Las líneas corresponden a la variedad morfológica del territorio pero también hacen referencia por un lado con su cromatismo a la diversidad paisajística (montañas altas, medias, valles y quebrados, lagos, ríos, termas, salinas, llanos y llanuras...) y por el otro al movimiento y acción que transmiten todas las actividades de turismo activo que se pueden practicar en toda la provincia. 
El punto representa tanto al sol y la alegría de sus gentes como el microclima que podemos disfrutar.

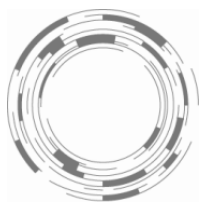

La Lente, hace alusión a la industria cinematográfica y las realidades paisajísitcas que podemos descubrir en San Luis como un escenario de cine. El turista interpone una lente entre su visión y la realidad con el fin de manipularla y crear arte, eso es Cine y el Cine es San Luis en acción. Pero además esos círculos concéntricos que conforman la lente con sus sombras responde a una estructura estudiada y matemática con el fin de hacer también una alusión al mundo de las ciencias y las nuevas tecnologías.

Con estas sencillas formas hemos transmitido la sofisticación, innovación y modernidad que requería la marca pero sin descuidar los elementos básicos de identidad como son los recursos naturales e hidrográficos de la provincia.

\section{El lema: San Luis en acción}

en acción es una coletilla que hemos decidido añadir a la marca para reforzar la idea de movimiento y turismo activo pero sobre todo por ser la palabra más pronunciada en la industria del cine (cámaras, luces, acción!). Por otro lado, hemos analizado sus posibles traducciones en inglés y son similares para evitar confusiones en los países emisores de habla inglesas.

5. Formulación del Plan de Reposicionamiento del destino turístico de San Luis, Argentina

\section{MISIÓN}


Posicionar a la provincia de San Luis como destino único, propio y de vanguardia que sea capaz de captar por si solo una importante afluencia turística.

\section{OBJETIVO GENERAL}

Creación de un producto turístico nuevo y diferenciado centrado en el cine, música aprovechando de las nuevas tecnologías que posee la provincia.

\section{ESTRATEGIAS}

\section{ESTRATEGIAS DE PRODUCTO}

Breve descripción del producto cinematográfico:

Se proporciona al visitante un servicio exclusivo, dinámico y flexible, dónde el turista cinéfilo decide en todo momento cómo, dónde y cuando vive su experiencia. Se trata de combinar los escenarios naturales, cultura y tecnología con el set cine. Esto será factible a través del centro de interpretación del cine.

\section{Aprovechar sinergias con otras marcas y colaboraciones 0} apadrinamientos de empresas notorias o personajes célebres del espectáculo.

- Coordinar con las otras marcas ya existentes en la provincia, por ejemplo la de alimentos, para trabajar en forma conjunta. Empleando la idea de la "marca paraguas". El objetivo es incluir el logo-marca de San Luis en todos los productos alimenticios envasados que se venden al resto del país y que se exporten al resto del mundo. Es una forma de lograr una mayor repercusión de la marca.

- Buscar apoyo de prescriptores activos.

- $\quad$ Actores y figuras celebres del mundo del espectáculo y deportes que deseen colaborar en campañas que promocionen el destino, ya sea a nivel nacional como extranjeros. Se pretende atraer a un personaje celebre que transmita la confianza necesaria para atraer a un determinado nicho de mercado. 
Realizar reality-documentales al estilo de la campaña que realizó Turismo de Perú para atraer a los turistas norteamericanos. En ese caso se utilizó un prescriptor, que es un prestigioso chef de la cocina neoyorquina.

Proporcionar nuevos usos turísticos al Set Cine de San Luís.

- Crear un Centro de Interpretación del Cine cuyo objetivo sea brindar un apoyo técnico al futuro turista para ayudar a comprender el funcionamiento de la industria cinematográfica, así como las técnicas y métodos para formar parte de esa industria.

- Creación del Taller-Cine móvil en el cual se ofrezca al visitante la posibilidad real de participar en el proceso de creación de una película, pudiendo elegir el mismo que papel desea desempeñar en la producción (desde actores, guionistas, cámara, etc) o siendo un mero espectador.

Lanzamiento de una marca de destino.

- Marca propuesta: "San Luís en acción”.

- $\quad$ Publicity y merchandising.

Aumentar y mejorar la repercusión de la industria cinematográfica de San Luís y dar notoriedad al Festival Internacional de cine de San Luís.

- Ampliar las relaciones públicas.

- $\quad$ Creación de Feria Interactiva de Cine en San Luis que coincida con la fecha de celebración del festival de cine.

- Taller de film hechos por el publico para campaña de promoción del destino (a través de concurso y castings).

PLANES DE ACCIÓN PARA LAS ESTRATEGIAS DE COMERCIALIZACIÓN Y COMUNICACIÓN

Captación de nuevos mercados interesados en el cine, música y nuevas tecnologías. 
Con respecto al desarrollo de ofertas especializadas e individualizadas resulta crucial realizar 2 tipos de segmentación del mercado: geográfico y sociodemográfico. Los cuales no son excluyentes, sino que incluso dependiendo de los diferentes mercados emisores se puede distinguir un perfil sociodemográfico muy concreto. Por ejemplo en el caso de Los Ángeles el nicho de mercado será principalmente un público más especializado en este producto.

\section{Segmentación según perfil geográfico (captación del mercado emisor)}

- A corto plazo captación de los mercados de Argentina, Brasil, EEUU (San Francisco, Los Ángeles, Nueva York) y España. Estos mercados han sido elegidos como prioritarios dado que los principales centros emisores de Argentina cuentan con un público interesado en la industria cinematográfica y en las tecnologías. El mercado brasileño es desde los últimos años el principal emisor de turistas a Argentina. Estados Unidos es un gran país emisor de turistas en la actualidad y cuenta con un mercado aficionado al cine. También se seleccionó a España para esta primera etapa debido a la relación estrecha que existe entre ambos países, en especial en el cine dónde ha habido un gran número de coproducciones.

- A medio plazo captación de mercados de Chile, México, Reino Unido, Francia, Holanda, Alemania e India.

- A largo plazo captación de mercados de Irlanda, resto de Estados Unidos, Canadá, Japón, Israel y China.

\section{Segmentación según perfil sociodemográfico:}

Este producto se puede adaptar a las exigencias de los siguientes perfiles de consumidores:

\begin{tabular}{lllllll}
\hline $\begin{array}{l}\text { Producto } \\
\text { Turístico }\end{array}$ & Segmento & Motivación y Comportamiento & & \\
\hline Tecnología & Familias con hijos & Mejora la situación económica, y & puede \\
y & mayores de 6 & & & & \\
\hline
\end{tabular}




\begin{tabular}{lll}
\hline $\begin{array}{l}\text { Cultura(cine } \\
\text { y música) }\end{array}$ & años. & despertar deseo de viajar. \\
& $\begin{array}{l}\text { Parejas jóvenes } \\
\text { sin hijos. }\end{array}$ & $\begin{array}{l}\text { Mayor poder adquisitivo, interés por } \\
\text { vacaciones e interés por las tecnologías. }\end{array}$ \\
& $\begin{array}{l}\text { Parejas de 45-65 } \\
\text { años. }\end{array}$ & $\begin{array}{l}\text { Buena situación económica, menos interés por } \\
\text { tecnologías, mayor interés por la cultura. }\end{array}$ \\
& $\begin{array}{l}\text { Estudiantes. } \\
\text { Soltero jóvenes. }\end{array}$ & $\begin{array}{l}\text { Poco poder adquisitivo, más aventureros y } \\
\text { mayor interés por la tecnología }\end{array}$ \\
\end{tabular}

Fuente: elaboración propia.

Creación de un Plan de Comunicación efectivo para mostrar la nueva imagen.

- $\quad$ Presentación de la marca San Luis a través de una rueda de prensa. El contacto con la prensa internacional será a través de INPROTUR.

- $\quad$ Presencia en prensa, medios especializados en viajes y cultura, y en las publicaciones de las compañías aéreas.

- Promover las relaciones públicas entre las principales empresas mayoristas del sector.

- Fam Trips a los mayoristas.

- $\quad$ Press Tour con los principales medios periodísticos especializados.

- $\quad$ Participación en las ferias, workshops (exclusivos para profesionales) y congresos más importantes de turismo (FITUR, Berlin, Miami, FIT, Brasil).

- Marketing de precisión: elaboración de campañas promocionales especificas para cada mercado a captar a corto plazo:

- Argentina:

- $\quad$ Cinebus (con propaganda de las jornadas y actividades).

- $\quad$ Publicidad en aeropuertos y metros.

- $\quad$ Carteles en paradas de Taxis.

- Brasil: 
atraer a las principales productoras de telenovelas a través de incentivos fiscales y económicos (el mercado brasileño es un gran consumidor de este tipo de series televisivas y está demostrado que las mismas influyen en la opinión del público).

Hermanamiento del Festival de cine de San Luis con el de

San Pablo.

○ España:

- realización de un documental en San Luis protagonizado por actores argentinos reconocidos en España (debido a la gran relación cinematográfica entre Argentina y España).

- Hermanamiento del Festival de cine de San Luis con el de San Sebastián.

- Estados Unidos:

- Hermanamiento del Festival de cine de San Luis con los de San Francisco y Los Ángeles.

Posicionamiento on-line.

- $\quad$ Desarrollo de web 2.0.

- $\quad$ Creación de Wikispace para el producto San Luis.

- Presencia de un Community Manager.

- Anunciar la página web de San Luís a través del Google Adwords (enlaces patrocinados). Para ello hay que seleccionar una serie de palabras y/o términos claves que están relacionados con el tema y la ubicación de la búsqueda. Se compra a Google estas palabras para crear enlaces comerciales y de este modo situarnos en los primeros tres puestos del rango de búsqueda de Google.

\section{Estrategia de fidelización de clientes}

- $\quad$ Creación de base de datos de nuestros clientes CRM.

- Segmentación de público con carné PLAY (visitantes interesados en el cine y tecnologías pero solamente como espectadores) y REC (visitantes activos, es decir aquellos que desean ser parte de una filmación o rodaje.)

- $\quad$ Programa de Fidelización "de su mirada a la pantalla" : 
Feria interactiva con talleres y jornadas de capacitación dentro de un recinto ferial existente en los principales centros emisores de Argentina (Buenos Aires, Córdoba y Rosario), dónde se participarán todos los implicados en la industria cinematográfica (directores, guionistas, actores, escenográfos, iluminadores, productores, camarógrafos, etc).

- Serán realizadas siempre durante la misma fecha con el objetivo de crear un flujo fiel de participantes.

\section{Estrategia de posicionamiento de San Luis como destino tecnológico.}

Además de posicionar San Luis como un destino cinematográfico, conviene también ampliar el horizonte hacia el desarrollo de las nuevas tecnologías, aprovechando el potencial que de por si ya tiene la provincia, pero además creando vínculos con grandes multinacionales del sector de las nuevas tecnologías. Se busca potenciar el desarrollo de la zona sur de san Luis que es la que menos está relacionada con el producto, una forma de dinamizar e incluirla en los circuitos turísticos.

- $\quad$ Formar parte de la Fundación de Google para entrar en su filosofía de la empresa. De este modo se pretende crear un vínculo entre el desarrollo tecnológico de San Luis y los avances en este campo que ofrece el imperio Google.

- Turismo espacial (existe una iniciativa por parte de los fundadores de Google en crear transbordadores espaciales para el turismo).

- Se propone inspirarse en el Evento "Burning Man" creado por Google, pero en el desierto del sur de San Luís. Consiste en crear parajes temporales estableciendo nuevos mundos, experimentando con el arte y la tecnología y se crea todo lo que una ciudad necesita (hippies tecnológicos).

\section{ESTRATEGIAS DE GESTIÓN Y PLANIFICACIÓN}

Fomentar la creación de un ente de gestión del turismo para San Luís que permita una cooperación y coordinación público-privada.

- Ente compuesto por asociaciones hoteleras y de empresas de turismo, profesores y alumnos de la universidad local, cámaras de turismo, asociaciones de la restauración y hostelería, la Film Commission de san Luis, el ministerio de 
Turismo de las Culturas y Deportes y demás agentes implicados en el sector. Para facilitar las labores de planificación y gestión del destino se elegirá al menos uno o dos representantes de cada grupo mencionado.

- $\quad$ Acuerdos publicitarios en los que interviene el sector público y privado.

- Llevar a cabo gestiones comerciales con compañías aéreas que vuelen a San Luís para promocionar San Luis en el fuselaje. Esto debe ser a través de un acuerdo entre el ente gestor, aeropuerto, compañías aéreas y gobierno.

- Fomentar la creación de un transporte turístico adecuado capaz de homogeneizar los principales hitos.

- $\quad$ Fomento de creación de incentivos económicos para permitir la creación de nuevas agencias de turismo receptor y mejoras de las existentes.

Fomentar la catalogación e inventario de bienes y servicios turísticos y colaborar en programas para desestacionalizar la demanda turística.

- Coordinación del proyecto de posicionamiento con los programas de turismo existentes del Ministerio de Turismo de San Luís, a nivel regional con el Plan de Dinamización Turístico de San Luís y también con planes nacionales.

Promover y fomentar propuestas legislativas de ordenación de la actividad turística y el desarrollo tecnológico, innovaciones, i+d.

- Programa para desarrollo tecnológico en hoteles, aplicaciones BlackBerry, i-phone; rutas a tiempo real.

- Estudio de nuevas tendencias en el sector hotelero como el modelo de los hoteles geek.

- Fomentar la nacionalización de la Universidad de La Punta y proporcionar notoriedad y prestigio a esta institución en los campos de la comunicación y audiovisual, investigación i+d para abrir vías de colaboración y cooperación con el Set Cine de San Luís.

Fomentar la mejora de la señalización turistica, la ampliación de puntos de información turística y la optimización de la calidad de bienes y servicios turísticos. 
- $\quad$ Colaboración en el diseño de nuevos centros de información completos in situ con folleteria clara y mapas precisos y actualizados.

- Colaboración en la mejora de gestión de los flujos turísticos en los aeropuertos existentes en la provincia.

Fomentar políticas medioambientales efectivas y de conservación del patrimonio.

- $\quad$ Sistema de control y seguimiento del impacto medioambiental causado por el turismo, mediante estudios con la colaboración del centro de investigación de la universidad y el apoyo de las cámaras de turismo.

- Promover la mejora del entorno sobre todo de la propia ciudad de San Luís.

\section{Conclusión}

El presente trabajo de investigación nace de la necesidad de la provincia argentina de San Luis de un reposicionamiento en el mercado turístico, que le permita competir con otros destinos de Argentina y de América Latina, creando un modelo que permita aplicar de manera conjunta y estructurada, entre el sector público y privado, las bondades que ofrecen las metodologías más usadas en la actualidad. La finalidad de este modelo es guiar a las organizaciones a implementar un sistema esbelto, que por ende, disminuya las debilidades y apoye a la competitividad de la provincia, haciéndola más productiva y alcanzando los índices de calidad exigidos por los turistas actuales.

Al inicio de este trabajo se determinó la necesidad de desarrollar una metodología como respuesta a la problemática existente, teniendo como objetivo el implementar un sistema que aprovechase los recursos de los que ya se disponían para dar a conocer la provincia al mundo.

Cuando comenzábamos a trabajar en el reposicionamiento de San Luis nos vimos muy emocionados. En el grupo de trabajo contábamos con dos argentinos, con una opinión ya formada de la provincia y que nos ayudaron 
mucho a entender algunos de los aspectos de estudio. A medida que avanzábamos con el trabajo nos dimos cuenta de que se trataba más de un trabajo de posicionamiento ya que San Luis no es una provincia conocida fuera de Argentina y dentro tiene una mala imagen asociada a la política.

Ante esta situación, desde la consultora, desarrollamos un modelo con nuestra respectiva metodología basada en las fortalezas que encontramos a lo largo del estudio de la provincia argentina de San Luis.

Finalmente hemos conseguido un producto turístico capaza de ofrecer un amplio abanico de posibilidades. Se han establecido 2 pilares fundamentales de actuación: cine y nuevas tecnologías, a través de los cuales se desglosa toda la oferta turística que puede llegar a brindar San Luis como un destino multifacético, novedoso e innovador. Estos 2 ejes actúan como motor impulsando el turismo de naturaleza, de termas, activo, cultural.

Determinadas estrategias y acciones que han sido enfocadas a un segmento 0 mercado especifico en el corto plazo, podrán ser utilizadas para abrirse a nuevos mercados. Esto se irá evaluando a medida que se vayan analizando los resultados de las estrategias a corto plazo.

La música como estrategia para captación de nuevos mercados se plantea a largo plazo. Citando el conocido refrán de "Quien mucho abarca poco aprieta", hemos creído conveniente dejar este foco de acción para el largo plazo, sin desecharlo, pues la provincia de San Luis cuenta en la actualidad con tecnología relacionada con la música (estudios de grabación modernos, sello discográfico propio) en la ciudad de Villa Mercedes. Una vez que el producto tecnológico planteado a corto plazo esté funcionando de un modo fluido, se deja la puerta abierta para considerar el añadido del mercado de la música, siguiendo en la línea de las artes y la tecnología.

\section{Fuentes de información y bibliografía}

Boullon, Roberto (2004), Marketin Turístrico: Una persepectiva desde la planificación (2 $\left.2^{\mathrm{a}} \mathrm{Ed}\right)$. Ed. Ediciones Turísticas, 2004.

Costa, J (2004), La imagen de marca, Paidós, Barcelona 
Trout, Jack y Rivkin, Steve (2001), Reposicionamiento: La estrategia competitiva en una era de hipercompetencia, cambio y crisis, Ed. Pirmaide

V.V.A.A (2009), The Times Universal Atlas of the World, Ed. Harpercollins Pub. V.V.A.A. (2006), Marketing de destinos turísticos. Ed. Editorial MAD, SL.

Fuentes de información:

www.welcomeargentina.com/sanluis/

www.argentinaturistica.com/sluiresenia.htm - Argentina

www.sanluis.gov.ar/

www.argentinaturismo.com.ar/sanluis/

www.ciudaddesanluis.gov.ar/

www.estadistica.sanluis.gov.ar

www.indec.mecon.ar/indec/indec dpe2 listado.asp

www.sanluisturismo.com.ar/ -

www.argentinaturistica.com/sluiresenia.htm - Argentina

guiahueney.blogspot.com

Para citar este artículo

Fernández Arcos, Alba - Cardoso Rolo, Carolina - López González, Ester - Falcón, Juan Pablo - Forbes Gudiño, Mavis Anet - Sánchez Canedo, Míriam - Pardo Neira, Soledad Trigo Rossier, Susana - Sánchez Canedo, Noemi (29-07-2011). PLAN DE REPOSICIONAMIENTO DEL DESTINO TURÍSTICO DE SAN LUIS, ARGENTINA.

REDMARKA - CIECID - Unidad de Investigación en Marketing Aplicado-Universidad de A

Coruña

Año III, Número 6, V1, pp.121-140

ISSN 1852-2300

URL del Documento: cienciared.com.ar/ra/doc.php? $\mathrm{n}=1495$

URL de la Revista: cienciared.com.ar/ra/revista. php?wid=39 